Original Article

\title{
Determinants of metabolic syndrome (MetS) amongst persons living in Dibrugarh District of Assam
}

\author{
Tulika Goswami Mahanta ${ }^{a,},{ }^{*}$ Rajnish Joshi ${ }^{b}$, Bhupendra Mahanta $^{c}$, \\ Pronab Gogoi ${ }^{d}$
}

${ }^{a}$ Associate Professor, Community Medicine, Assam Medical College, Dibrugarh, India

${ }^{\mathrm{b}}$ Assistant Professor, Medicine, AIIMS, Bhopal, India

${ }^{\mathrm{c}}$ Associate Professor, Medicine, Assam Medical College, Dibrugarh, India

d Statistician, Assam Medical College, Dibrugarh, India

\section{A R T I C L E I N F O}

Article history:

Received 25 November 2016

Accepted 22 December 2016

Available online 6 January 2017

\section{Keywords:}

Metabolic syndrome

Dibrugarh

Assam

Risk factor

Cardiovascular diseases

\begin{abstract}
A B S T R A C T
Problem considered: As India is considered as the diabetic capital of world, a huge burden of undiagnosed metabolic syndrome (MetS) is a possibility. Early intervention can be planned if MetS can be detected early to avert cardiovascular morbidities.

Aims: To assess determinants of MetS amongst population living in Dibrugarh District of Assam.

Methods: Community based cross-sectional study conducted in Dibrugarh District of Assam, with multistep sampling. Study area, i.e., four rural sub-centres and two urban electoral blocks, were selected randomly. From the list of population of selected area the consenting eligible were included. Sample size was 1700 population with MetS. Socio-demographic information, World Health Organization's Steps questionnaire for behavioural risk factors along with dietary history, anthropometric assessment and laboratory investigations were conducted in three stages. Statistical analysis used: rates, ratio, proportion, univariate and multivariate analysis.

Results: MetS was $47.6 \%$ (1606 of 3372 screened) and mean age $47.1 \pm 10.9$ years. Univariate analysis showed educational status, occupation, family history of hypertension and diabetes, tobacco and alcohol consumption, financial stress, depression, less physical activity, more consumption of meat, fish, egg, diary products, and high-energy food significantly associated with MetS. Multiple logistic regression analysis showed age, education, occupation, computer owner (OR: 3.69, CI: $2.10-6.47, p=0.000$ ), family history of diabetes (OR: 3.18 , CI: $2.10-6.47, p=0.000$ ), stress (OR: 1.85 , CI: $1.45-2.35, p=0.000$ ), consuming dairy product $\geq 3$ times/week (OR: 2.57, CI: 1.68-3.94, $p=0.000$ ) and nuts and oilseed <3 times/week (OR: 2.37, CI: $1.90-2.96, p=0.000$ ) have significant association.
\end{abstract}

\footnotetext{
* Corresponding author at: Community Medicine, Assam Medical College, Dibrugarh 786001, India. Tel.: +91 9435032539.

E-mail address: drtulikagoswami@gmail.com (T.G. Mahanta).

http://dx.doi.org/10.1016/j.cegh.2016.12.004

2213-3984/@ 2016 INDIACLEN. Published by Elsevier, a division of RELX India, Pvt. Ltd. All rights reserved.
} 
Conclusions: Risk factors of cardiovascular diseases were rampant amongst persons with

MetS. Screening for risk factors amongst them can give opportunity for early intervention.

@ 2016 INDIACLEN. Published by Elsevier, a division of RELX India, Pvt. Ltd. All rights

reserved.

\section{Introduction}

Type 2 diabetes mellitus (T2DM), is a heterogeneous disease with strong genetic component, characterized by insulin resistance and impaired beta-cell function. Combination of insulin resistance and compensatory hyper-insulinemia increases risk of hypertension and dyslipidaemia characterized by high plasma triglycerides (TG) and low high-density lipoprotein cholesterol (HDL-C) concentration. These changes increase risk of cardiovascular disease (CVD). This cluster of related abnormalities was previously designated as Syndrome $\mathrm{X}^{1}$ The increased risk for developing CVD and diabetes associated with a cluster of metabolic abnormalities, is referred to as metabolic syndrome (MetS). MetS includes hypertension, glucose intolerance, high TG, low HDL-C and abdominal obesity. ${ }^{2}$ Strong association of abdominal obesity with metabolic abnormalities has prompted the National Institute of Health (NIH) of the United States of America and the World Health Organization (WHO) to issue guidelines for use of gender-specific waist circumference (WC) cut points to identify abdominal obesity. ${ }^{3}$

MetS was diagnosed based on modified ATP III guidelines if any three of the following abnormalities were present: abdominal obesity (defined as WC $\geq 90 \mathrm{~cm}$ for men and $\geq 80 \mathrm{~cm}$ for women, according to modified Asia Pacific World Health Organization guidelines), high blood pressure (systolic blood pressure [sBP] $\geq 130 \mathrm{mmHg}$, diastolic blood pressure [dBP] $\geq 85 \mathrm{mmHg}$, or who self-reported hypertension and on anti-hypertensives), hypertriglyceridemia or low HDL cholesterol. Obesity was defined as body mass index (BMI) $\geq 25 \mathrm{~kg} / \mathrm{m}^{2}$ according to modified Asia Pacific World Health Organization guidelines. $^{4,5}$

MetS was associated with increased risk for T2DM and CVDs. However, different diagnostic criteria have been recommended by different expert groups. Study shows high prevalence of hypertension amongst tea garden population of Dibrugarh. ${ }^{6}$ Therefore, we need to study MetS among these population. Individuals with MetS are associated with approximately five and two-fold increased risk for T2DM and CVD respectively and risk factor for all-cause mortality. ${ }^{7,8}$ MetS has been linked with obesity and sedentary lifestyle, both are modifiable. ${ }^{9}$ More effort to promote a healthy lifestyle with increased physical activity (PA) and reduced obesity was needed. ${ }^{10,11}$ Individuals with MetS should be identified early so that their cardiovascular risk factors can be reduced. ${ }^{12}$ Definitions of MetS are changing (ATP-III/IDF etc.) based on perceived risk factors. India, considered as diabetic capital, sharing huge burden of undiagnosed MetS is a possibility and therefore present study is planned to estimate the determinant of MetS.

\section{Methods}

\subsection{Setting}

Dibrugarh District of Assam and is at the northern-most tip of the state, situated on the banks of River Brahmaputra, spread over $3000 \mathrm{~km}^{2}$, and is populated by about 1.2 million individuals (0.9 million rural, 0.3 million urban). There are 1362 villages under 231 health sub-centres. In urban areas there were 96 electoral blocks. ${ }^{13}$

\subsection{Ethics statement}

Institutional ethics committee approval from Assam Medical College and necessary permissions was obtained from Joint Director of Health Services and the Assam Branch of the Indian Tea Association. Written informed consent, sought from all eligible, and those consenting, included. If a participant could not read or write, verbal information was provided, and consent was recorded as thumb impression in presence of two impartial witnesses. Surveys were preceded by meetings with community leaders.

\subsection{Subject selection}

For detecting MetS, after overnight fast (10-12 h), blood samples was obtained for determination of plasma glucose, plasma HDL-C and plasma TG levels. Modified ATP III guidelines was used for identification of persons with MetS. ${ }^{4}$ A screening log was prepared by house to house survey and all eligible individuals were enrolled. Laboratory investigation was done for all eligible and those fulfilling criteria of MetS were included till the required sample size was achieved.

The sample size was 1546, calculated by taking prevalence of MetS as 19.9 and $95 \%$ CI and relative precision 10\%. Taking $10 \%$ non-response rate and rounding up, sample size inflated to 1700 .

\subsection{Sampling design}

Cross-sectional study with multistep sampling to include representative population. Initially, sub-centres was enlisted from which four rural and two urban electoral block were selected using computerized random numbers. From the list of eligible population of selected sub centre area the required sample of population was enrolled (1700; 1400 rural, 300 urban). Then, household survey was done to enlist all eligible consenting individual aged 20-60 years. Socio-demographic, behavioural risk factors, medical history and family history of diabetes were assessed using WHOs Steps questionnaire. 
Dietary history was taken using food frequency questionnaire and 24-hour recall. Anthropometric assessment and laboratory investigations were conducted. All consenting adults of selected areas having MetS were considered eligible for inclusion. Measurement of height, weight and WC was done. Height and weight was measured to nearest $0.5 \mathrm{~cm}$ and $0.1 \mathrm{~kg}$, respectively. BMI was calculated as weight $(\mathrm{kg})$ divided by height $(\mathrm{m})$ squared. WC, determined at umbilical level $(\mathrm{cm})$ to nearest $0.1 \mathrm{~cm}$ using a measuring tape positioned at midpoint between lowest rib and iliac crest. Similarly hip circumference was taken around widest portion of buttocks as recommended by WHO STEP wise Approach to Surveillance (STEPS).

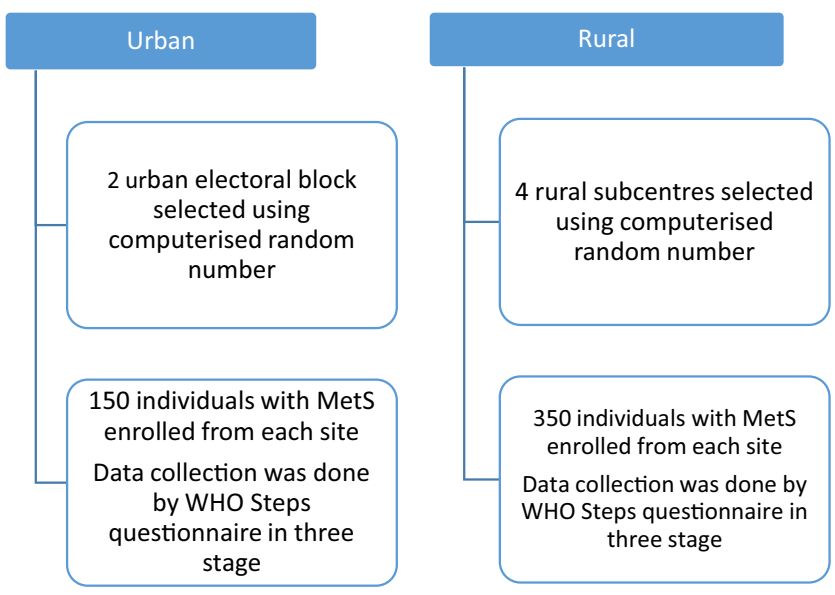

\subsection{Definition of metabolic syndrome}

MetS was defined by modified NCEP ATP III and IDF groups. ${ }^{4}$ Presence of any three of five factors is required for diagnosis of MetS: abdominal obesity, hyper-triglyceridemia (TG $\geq 1.7 \mathrm{mmol} / \mathrm{L}$ ); low HDL cholesterol (HDL cholesterol $\leq 1.03 \mathrm{mmol} / \mathrm{L}$ for men and $\leq 1.29 \mathrm{mmol} / \mathrm{L}$ for women); elevated blood pressure (sBP $\geq 130 \mathrm{mmHg}$ and/or $\mathrm{dBP} \geq 85 \mathrm{mmHg}$ or current use of antihypertensive drugs); impaired fasting glucose (fasting plasma glucose $\geq 5.6 \mathrm{mmol} / \mathrm{L}$ ). Modified NCEP ATP III criteria suggested cut-off points of WC should be ethnic specific where individuals of Asian origin should use cut-off of $90 \mathrm{~cm}$ in men and $80 \mathrm{~cm}$ in women.

\subsection{Biochemical measurements}

Measurement of plasma glucose was carried out by glucose oxidase method, using a commercial enzymatic reagent (Ozone biochemical Kit). Plasma TG and cholesterol concentrations were measured by enzymatic methods (Lifescan Kit).

\subsection{Qualitative study}

FGD and in-depth interview (IDI) of persons having MetS was done - one FGD and 5 IDI in each study area to understand, barriers affecting care seeking behaviour and compliance of treatment and to assess quality of care for CVD.

\subsection{Statistical analysis}

Most variables, collected as continuous measures and standard definitions, were used for categorical classifications. Information about each risk factor, described for, entire population. Student's t-test was used for continuous variables, and chi square test for dichotomous variables. Statistical analysis was done using SPSS software.

\section{Results}

Enrolment was 3372 to achieve required sample size. Mean age of study participants was $47.1 \pm 10.9$ years. Prevalence of MetS was $47.6 \%$, which was more common amongst men than women (53.3\% vs. $51.1 \%$ ). MetS was higher amongst welleducated people. Prevalence of MetS was found to be higher amongst house makers (88.3\%), business persons (71.1\%), and government service holder (70.3\%). Those staying in their own home, have less MetS compared to those staying in rented house $(76.4 \%$ vs. $44.6 \%)$. Similarly, those having their own vehicle or motor cycle are having MetS more than those not having ( $73.6 \%$ vs. $45.8 \%$ and $67.2 \%$ vs. $38.5 \%$ subsequently). Those owning a computer were having higher prevalence of MetS than those not having ( $86.1 \%$ vs. $45.4 \%$ ).

Overall prevalence of hypertension was $17 \%$. Hypertension prevalence was $62.2 \%$ amongst person having MetS. Overall prevalence of diabetes was $5.7 \%$ and amongst MetS, it was $89.1 \%$, indicating very high prevalence of diabetes amongst those having MetS. Other cardiovascular symptoms like angina was found in $3.6 \%$ of which $52.5 \%$ were having MetS. History of myocardial infarction was found in $0.3 \%$, of which $60 \%$ were having MetS. Similarly stroke was found in $0.5 \%$, of which $88.9 \%$ were having MetS (Table 1).

Behavioural risk factor distribution - current tobacco user was $40.6 \%$ (774), while $47.2 \%$ (102) were former user of tobacco. Amongst studied participants having MetS, 38.1\% (619) consumes alcohol. Financial stress was moderate or severe in $44.4 \%$ (748) cases, while $50.9 \%$ (413) had stress for several period or permanent stress. Episodes of depression were present in last year in $60.9 \%$ (313) cases. Majority in work place $(55.4 \%$, 382) were mainly sedentary, while $47.2 \%$ (375) walks, and $37.7 \%$ (228) does heavy PA. During leisure time also $62.1 \%$ (425) were sedentary, while only $41.3 \%$ does moderate exercise (multiple response included) (Table 2).

Univariate analysis showing religion [Hindu (OR: 2.20, CI: 1.63-2.96, $p=0.000$ ), Islam (OR: 2.13 , CI: $1.36-3.33, p=0.001$ )] compared to Christian, educational status [I-VIII standard (OR: 1.55, CI: 1.29-1.87, $p=0.000$ ), IX-XII standard (OR: 3.34, CI: 2.81$3.98, p=0.000$ ), college/university (OR: 3.24, CI: 2.56-4.11, $p=0.000)$ ] compared to illiterate, marital status [unmarried (OR: 2.25, CI: 1.59-3.17, $p=0.000$ ), married (OR: 1.06, CI: 0.881.28, $p=0.500$ )], occupation [farmer (OR: 4.85, CI: 3.45-6.80, $p=0.000$ ), house maker (OR: 14.92 , CI: 11.43-19.46, $p=0.000$ ), business (OR: 2.01, CI: 1.32-3.04, $p=0.001$ ), government service (OR: 4.74, CI: 3.52-6.40, $p=0.000$ ), self-employed (OR: 4.59, CI: 3.52-6.40, $p=0.000)$ ], motorcycle owner [OR: 3.26, CI: 2.80-3.80, $p=0.000$ ], land/property owner [OR: 1.98, CI: 1.64-2.40, $p=0.000$ ] were having significant association with MetS. Hindu and Islam believers were having more MetS than Christians. 
Table 1 - Cardiovascular diseases amongst person having metabolic syndrome (MetS).

\begin{tabular}{|c|c|c|c|}
\hline \multirow[t]{2}{*}{ Cardiovascular disease } & \multicolumn{2}{|c|}{ Metabolic syndrome } & \multirow[t]{2}{*}{ Total, $n(\%)$} \\
\hline & No, $n(\%)$ & Yes, $n(\%)$ & \\
\hline \multicolumn{4}{|l|}{ Hypertension } \\
\hline Yes & $217(37.8)$ & $357(62.2)$ & $574(17.0)$ \\
\hline No & $1549(55.4)$ & $1248(44.6)$ & $2797(83.0)$ \\
\hline \multicolumn{4}{|l|}{ Diabetes } \\
\hline Yes & 21 (10.9) & $172(89.1)$ & $193(5.7)$ \\
\hline No & $1745(54.9)$ & $1433(45.1)$ & $3178(94.3)$ \\
\hline \multicolumn{4}{|l|}{ Angina } \\
\hline Yes & $58(47.5)$ & $64(52.5)$ & $122(3.6)$ \\
\hline No & $1707(52.6)$ & $1539(47.4)$ & $3246(96.4)$ \\
\hline \multicolumn{4}{|l|}{ Myocardial infraction (MI) } \\
\hline Yes & $4(40.0)$ & $6(60.0)$ & $10(0.3)$ \\
\hline No & $1762(52.4)$ & $1600(47.6)$ & 3362 (99.7) \\
\hline \multicolumn{4}{|l|}{ Stroke } \\
\hline Yes & $2(11.1)$ & 16 (88.9) & $18(0.5)$ \\
\hline No & $1764(52.6)$ & $1590(47.4)$ & 3354 (99.5) \\
\hline \multicolumn{4}{|l|}{ Other vascular diseases } \\
\hline Yes & $3(33.3)$ & $6(66.7)$ & $9(0.3)$ \\
\hline No & $1763(52.4)$ & $1600(47.6)$ & $3363(99.7)$ \\
\hline
\end{tabular}

Higher the education level more is the MetS occurrence. Those acquiring different assets like for example computer [OR: 7.45, CI: 4.89-11.34, $p=0.000$ ] were more vulnerable to MetS, indicating higher socio-economic status having more MetS. Family history of hypertension [OR: 1.51, CI: 1.23-1.85, $p=0.000$ ] and diabetes [OR: 4.12, CI: 3.23, $p=0.000$ ] also show significant association with MetS (Table 3).
Tobacco consumption [former user (OR: 0.63, CI: 0.47-0.85, $p=0.002$ ) and current user (OR: 0.48, CI: 0.42-0.56, $p=0.000$ )], alcohol consumption [OR: 0.49, CI: 0.43-0.57, $p=0.000$ ], financial stress [OR: $0.49, \mathrm{CI}: 0.43-0.57, p=0.000]$, depression [OR: 1.88 , CI: $1.55-2.28, p=0.000$ ] and less PA [moderate PA (OR: 1.51 , CI: $1.24-1.84, p=0.000$ ), mainly sedentary (OR: 1.74 , CI: $1.43-$ $2.11, p=0.000)$ ] were significantly associated with MetS. Three

\section{Table 2 - Behavioural risk factor distribution amongst studied population.}

\begin{tabular}{|c|c|c|c|}
\hline \multirow[t]{2}{*}{ Behavioural profile } & \multicolumn{2}{|c|}{ Metabolic syndrome } & \multirow[t]{2}{*}{ Total, $n(\%)$} \\
\hline & No, $n(\%)$ & Yes, $n(\%)$ & \\
\hline \multicolumn{4}{|l|}{ Tobacco user } \\
\hline Formerly used & $114(52.8)$ & $102(47.2)$ & $216(6.4)$ \\
\hline Currently user & $1131(59.4)$ & $774(40.6)$ & $1905(56.5)$ \\
\hline Never used & $521(41.6)$ & $730(58.4)$ & $1251(37.1)$ \\
\hline \multicolumn{4}{|l|}{ Consumed alcohol } \\
\hline Yes & 929 (61.9) & $572(38.1)$ & $1501(44.5)$ \\
\hline No & $837(44.7)$ & $1034(55.3)$ & $1871(55.5)$ \\
\hline \multicolumn{4}{|l|}{ Financial stress } \\
\hline Little or none & $831(49.2)$ & $858(50.8)$ & $1689(50.1)$ \\
\hline Moderate or severe & $935(55.6)$ & $748(44.4)$ & $1638(49.9)$ \\
\hline \multicolumn{4}{|l|}{ Felt stress in last year } \\
\hline Never or some periods & 1367 (53.4) & 1193 (46.6) & $2560(75.9)$ \\
\hline Several periods or permanent stress & $399(49.1)$ & $413(50.9)$ & $812(24.1)$ \\
\hline \multicolumn{4}{|c|}{$\begin{array}{l}\text { Past } 12 \text { months, was ever you felt sad, blue or depressed } \\
\text { for } 2 \text { weeks or more in a row }\end{array}$} \\
\hline Yes & $201(39.1)$ & $313(60.9)$ & $514(15.2)$ \\
\hline No & $1565(54.8)$ & $1293(45.2)$ & $2858(84.8)$ \\
\hline \multicolumn{4}{|l|}{ Active at work } \\
\hline Mainly sedentary & $307(44.6)$ & $382(55.4)$ & $689(20.4)$ \\
\hline Predominately walking & $420(52.8)$ & $375(47.2)$ & $795(23.6)$ \\
\hline Mainly walking & $232(50.9)$ & $224(49.1)$ & $456(13.5)$ \\
\hline Heavy physical labour & $376(62.3)$ & $228(37.7)$ & 604 (17.9) \\
\hline Subject does not work & $431(52.1)$ & $397(47.9)$ & $828(24.6)$ \\
\hline \multicolumn{4}{|l|}{ During leisure time } \\
\hline Mainly sedentary & 259 (37.9) & $425(62.1)$ & $684(20.3)$ \\
\hline Mild exercise & $398(53.6)$ & $344(46.4)$ & $742(22.0)$ \\
\hline Moderate exercise & $1104(58.7)$ & $778(41.3)$ & $1882(55.8)$ \\
\hline Strenuous exercise & $5(7.8)$ & $59(92.2)$ & $64(1.9)$ \\
\hline
\end{tabular}


Table 3 - Univariate logistic regression analysis of socio-demographic variables with MetS.

\begin{tabular}{|c|c|c|c|c|c|}
\hline Variable & $N(\%)$ & PR (\%) & OR & $95 \% \mathrm{CI}$ & $p$-value \\
\hline \multicolumn{6}{|l|}{ Age group } \\
\hline $20-29$ & $46(1.4)$ & $19(41.3)$ & Ref. & & \\
\hline $30-39$ & $893(26.5)$ & $353(39.5)$ & 0.92 & $0.51-1.69$ & 0.810 \\
\hline $40-49$ & $1208(35.8)$ & $576(47.7)$ & 1.29 & $0.71-2.35$ & 0.396 \\
\hline $50-59$ & $665(19.7)$ & $378(56.8)$ & 1.87 & $1.02-3.43$ & 0.043 \\
\hline $60 \&$ above & 560 (16.6) & $280(50.0)$ & 1.42 & $0.77-2.61$ & 0.259 \\
\hline \multicolumn{6}{|l|}{ Religion } \\
\hline Christian & $218(6.5)$ & $66(30.3)$ & Ref. & & \\
\hline Hindu & $3021(89.6)$ & $1476(48.9)$ & 2.20 & $1.63-2.96$ & 0.000 \\
\hline Islam & $133(3.9)$ & $64(48.1)$ & 2.13 & $1.36-3.33$ & 0.001 \\
\hline \multicolumn{6}{|l|}{ Gender } \\
\hline Male & $1778(52.7)$ & $827(46.5)$ & Ref. & & \\
\hline Female & $1594(47.3)$ & 779 (48.9) & 1.09 & $0.96-1.25$ & 0.171 \\
\hline \multicolumn{6}{|l|}{ Education status } \\
\hline None & $1396(41.4)$ & $479(34.3)$ & Ref. & & \\
\hline 1-8 standard & $678(20.1)$ & $304(44.8)$ & 1.55 & $1.29-1.87$ & 0.000 \\
\hline 9-12 standard & 915 (27.1) & $582(63.6)$ & 3.34 & $2.81-3.98$ & 0.000 \\
\hline College/university & $383(11.4)$ & $241(62.9)$ & 3.24 & $2.56-4.11$ & 0.000 \\
\hline \multicolumn{6}{|l|}{ Marital status } \\
\hline Widowed/separated & $539(16.0)$ & $243(45.3)$ & Ref. & & \\
\hline Unmarried & $189(5.6)$ & $123(65.1)$ & 2.25 & $1.59-3.17$ & 0.000 \\
\hline Married & $2644(78.4)$ & $1239(46.9)$ & 1.06 & $0.88-1.28$ & 0.500 \\
\hline \multicolumn{6}{|l|}{ Occupation } \\
\hline Skilled labour & $1152(34.2)$ & $388(33.7)$ & Ref. & & \\
\hline Farmer & $97(2.9)$ & 49 (50.5) & 2.01 & $1.32-3.04$ & 0.001 \\
\hline House maker & 669 (19.8) & $591(88.3)$ & 14.92 & $11.43-19.46$ & 0.000 \\
\hline Business & $187(5.5)$ & $133(71.1)$ & 4.85 & $3.45-6.80$ & 0.000 \\
\hline Govt. service & $249(7.4)$ & $176(70.7)$ & 4.74 & $3.52-6.40$ & 0.000 \\
\hline Self employed & $60(1.8)$ & $42(70.0)$ & 4.59 & $2.61-8.08$ & 0.000 \\
\hline Other & $958(28.4)$ & $227(23.7)$ & 0.61 & $0.50-0.74$ & 0.000 \\
\hline \multicolumn{6}{|l|}{ Car } \\
\hline No & $3152(93.5)$ & $1444(45.8)$ & Ref. & & \\
\hline Yes & $220(6.5)$ & $162(73.6)$ & 3.30 & $2.42-4.49$ & 0.000 \\
\hline \multicolumn{6}{|l|}{ Motorcycle } \\
\hline No & $2297(68.1)$ & $884(38.5)$ & Ref. & & \\
\hline Yes & $1075(31.9)$ & $722(67.2)$ & 3.26 & $2.80-3.80$ & 0.000 \\
\hline \multicolumn{6}{|l|}{ Television } \\
\hline No & $656(19.5)$ & $284(43.3)$ & Ref. & & \\
\hline Yes & $2716(80.5)$ & $1322(48.7)$ & 1.24 & $1.04-1.47$ & 0.013 \\
\hline \multicolumn{6}{|l|}{ Other land/property } \\
\hline No & $2847(84.4)$ & $1281(45.0)$ & Ref. & & \\
\hline Yes & $525(15.6)$ & $325(61.9)$ & 1.98 & $1.64-2.40$ & 0.000 \\
\hline \multicolumn{6}{|l|}{ Computer } \\
\hline No & $3185(94.5)$ & $1445(45.4)$ & Ref. & & \\
\hline Yes & $187(5.5)$ & $161(86.1)$ & 7.45 & $4.89-11.34$ & 0.000 \\
\hline \multicolumn{6}{|c|}{ Hypertension (family history) } \\
\hline No & $2941(87.2)$ & $1362(46.3)$ & Ref. & & \\
\hline Yes & $431(12.8)$ & $244(56.6)$ & 1.51 & $1.23-1.85$ & 0.000 \\
\hline \multicolumn{6}{|l|}{ Diabetes (family history) } \\
\hline No & $2972(88.1)$ & $1301(43.8)$ & Ref. & & \\
\hline Yes & $400(11.9)$ & $305(76.2)$ & 4.12 & $3.23-5.25$ & 0.000 \\
\hline
\end{tabular}

times or more consumption of meat [OR: 2.72 , CI: 2.01-3.67, $p=0.000$ ], fish [OR: 1.31, CI: 1.13-1.52, $p=0.000]$, egg [OR: 2.55, CI: $1.90-3.42, p=0.000]$, diary product [OR: 4.08 , CI: 2.96-5.62, $p=0.000$ ], high energy food [OR: $2.29, \mathrm{CI}: 1.77-2.98, p=0.000]$, sweets/deserts [OR: 1.75 , CI: 1.39-2.19, $p=0.000$ ] and less nuts and oilseed consumption [OR: 1.61, CI: 1.37-1.87, $p=0.000$ ] were significantly associated with MetS (Table 4).

Multiple logistic regression analysis shows increasing age [30-39 years (OR: 12.83, CI: 5.61-29.34, $p=0.000), 40-49$ years (OR: 18.56, CI: 8.11-42.45, $p=0.000$ ), 50-59 years (OR: 26.65, CI: 11.49 $61.80, p=0.000$ ), 60 years and above (OR: 37.74 , CI: 15.97-89.16, $p=0.000)$ ] in comparison with 20-29 years, education status [1-8 standard (OR: 1.38, CI: 1.08-1.78), 9-12 years (OR: 2.95, CI: 2.25-3.86, $p=0.000$ ), college/university (OR: 3.44, CI: 2.33-5.05, $p=0.000)$ ] in comparison with illiteracy, occupation [house maker (OR: 15.24, CI: 10.73-21.65, $p=0.000$ ), business (OR: 1.83, CI: $1.20-2.81, p=0.005$ ), government service (OR: 2.38 , CI: 1.54 3.66, $p=0.000)$ ] compared to skilled worker, computer owner (OR: 3.69, CI: 2.10-6.47, $p=0.000$ ), family history of diabetes (OR: 3.18, CI: 2.10-6.47, $p=0.000)$, feeling stressed in last year (OR: 1.85, CI: $1.45-2.35, p=0.000$ ), moderate PA (OR: 1.45 , CI: 1.11-1.90, $p=0.006$ ) compared to heavy PA, consuming dairy 
Table 4 - Univariate logistic regression analysis of behavioural risk factors with MetS.

\begin{tabular}{|c|c|c|c|c|c|}
\hline Behavioural profile & $N(\%)$ & PR (\%) & OR & $95 \% \mathrm{CI}$ & $p$-value \\
\hline \multicolumn{6}{|l|}{ Tobacco user } \\
\hline Never used & $1251(37.1)$ & $730(58.4)$ & Ref. & & \\
\hline Formerly used & $216(6.4)$ & $102(47.2)$ & 0.63 & $0.47-0.85$ & 0.002 \\
\hline Currently user & $1905(56.5)$ & $774(40.6)$ & 0.48 & $0.42-0.56$ & 0.000 \\
\hline \multicolumn{6}{|l|}{ Consumed alcohol } \\
\hline No & $1871(55.5)$ & $1034(55.3)$ & Ref. & & \\
\hline Yes & $1501(44.5)$ & $572(38.1)$ & 0.49 & $0.43-0.57$ & 0.000 \\
\hline \multicolumn{6}{|l|}{ Financial stress } \\
\hline Moderate or severe & $1638(49.9)$ & $748(44.4)$ & Ref. & & \\
\hline Little or none & $1689(50.1)$ & $858(50.8)$ & 1.29 & $1.12-1.47$ & 0.000 \\
\hline \multicolumn{6}{|l|}{ Felt stress in last year } \\
\hline Never or some periods & $2560(75.9)$ & $1193(46.6)$ & Ref. & & \\
\hline Several periods or permanent stress & $812(24.1)$ & $413(50.9)$ & 1.18 & $1.01-1.38$ & 0.034 \\
\hline \multicolumn{6}{|c|}{$\begin{array}{l}\text { Past } 12 \text { months, was ever you felt sad, blue or depressed } \\
\text { for } 2 \text { weeks or more in a row }\end{array}$} \\
\hline No & $2858(84.8)$ & $1293(45.2)$ & Ref. & & \\
\hline Yes & $514(15.2)$ & $313(60.9)$ & 1.88 & $1.55-2.28$ & 0.000 \\
\hline \multicolumn{6}{|l|}{ Active at work } \\
\hline Heavy physical labour & $604(17.9)$ & $228(37.7)$ & Ref. & & \\
\hline Moderate work & $1251(37.1)$ & $599(47.9)$ & 1.51 & $1.24-1.84$ & 0.000 \\
\hline Subject does not work & $1517(45.0)$ & 779 (51.4) & 1.74 & $1.43-2.11$ & 0.000 \\
\hline \multicolumn{6}{|l|}{ Meat } \\
\hline$<3$ times/week & 3159 (93.7) & $1457(46.1)$ & Ref. & & \\
\hline$\geq 3$ times/week & $213(6.3)$ & $149(70.0)$ & 2.72 & $2.01-3.67$ & 0.000 \\
\hline \multicolumn{6}{|l|}{ Fish } \\
\hline$<3$ times/week & $2305(68.4)$ & $1048(45.5)$ & Ref. & & \\
\hline$\geq 3$ times/week & $1067(31.6)$ & $558(52.3)$ & 1.31 & $1.13-1.52$ & 0.000 \\
\hline \multicolumn{6}{|l|}{ Egg } \\
\hline$<3$ times/week & $3152(93.5)$ & $1455(46.2)$ & Ref. & & \\
\hline$\geq 3$ times/week & $220(6.5)$ & $151(68.6)$ & 2.55 & $1.90-3.42$ & 0.000 \\
\hline \multicolumn{6}{|l|}{ Dairy products } \\
\hline$<3$ times/week & 3147 (93.3) & $1432(45.5)$ & Ref. & & \\
\hline$\geq 3$ times/week & $225(6.7)$ & $174(77.3)$ & 4.08 & $2.96-5.62$ & 0.000 \\
\hline \multicolumn{6}{|l|}{ High energy food } \\
\hline$<3$ times/week & 3097 (91.8) & $1424(46.0)$ & Ref. & & \\
\hline$\geq 3$ times/week & $275(8.2)$ & $182(66.2)$ & 2.29 & $1.77-2.98$ & 0.000 \\
\hline \multicolumn{6}{|l|}{ Desserts/sweet } \\
\hline$<3$ times/week & 3029 (89.8) & $1400(46.2)$ & Ref. & & \\
\hline$\geq 3$ times/week & $343(10.2)$ & $206(60.1)$ & 1.75 & $1.39-2.19$ & 0.000 \\
\hline \multicolumn{6}{|l|}{ Nuts/seeds } \\
\hline$\geq 3$ times/week & $934(27.7)$ & 366 (39.2) & Ref. & & \\
\hline$<3$ times/week & $2438(72.3)$ & $1240(50.9)$ & 1.61 & $1.37-1.87$ & 0.000 \\
\hline
\end{tabular}

product $\geq 3$ times/week (OR: 2.57, CI: $1.68-3.94, p=0.000$ ) and nuts and oilseed $<3$ times/week (OR: 2.37, CI: 1.90-2.96, $p=0.000$ ) significantly associated with MetS (Table 5).

\subsection{Results of qualitative study}

Six FGD including 52 individuals having MetS and 27 in-depth interviews were conducted during the study. The results are as follows.

Health care seeking behaviour of persons with Mets: Majority sought health care if they feel any problems (84.8\%), while few (15.2\%) do not avail timely consultation for any health problem. Fact that ailment reaches its most severe form owe to several factors, encompassing both individual and societal parameters. Following points encompass some of the issues: people have tendency to ignore one's symptoms and pass it as common illness. Prevalence of other forms of treatment (homoeopathic, Ayurvedic, local/herbal medicines, yoga, witch doctors), little faith in prevalent form of treatment, monetary issues, misinformation about ailments/ treatments (medicines will not cure it and only suppress it, there might be severe side effects to medicine, medicines might damage vital organs like kidneys), prior bad experience etc. Many feels that medicine for hypertension should not be taken daily as it may further lower BP, or they may become dependent to it. "Anti-hypertensive should be taken only when BP goes up" was common believe in community (Fig. 1).

Health care preference: Most (78.8\%) preferred allopathic medicine. While Ayurvedic treatment was sought by $14.7 \%$, 6.5\% sought homoeopathic treatment.

Reasons for seeking healthcare: Majority (75.7\%) sought healthcare for early recovery, while $17.6 \%$ sought to keep track of health care and $6.7 \%$ seek as family members force to seek.

Reasons for not seeking health care: Majority (71.8\%) did not seek health care thinking it as minor illness. While $12.7 \%$ could not seek health care due to distance to health facility, 9.2\% think the consultation is too expensive, $3.6 \%$ think that 
Table 5 - Multivariate logistic regression analysis with adjusted odds ratio and $\mathbf{9 5 \%}$ CI.

\begin{tabular}{|c|c|c|c|c|c|}
\hline Variable & $N(\%)$ & PR (\%) & AOR & $95 \% \mathrm{CI}$ & $p$-value \\
\hline \multicolumn{6}{|l|}{ Age group } \\
\hline $20-29$ & $46(1.4)$ & $19(41.3)$ & Ref. & & \\
\hline $30-39$ & $893(26.5)$ & $353(39.5)$ & 12.83 & $5.61-29.34$ & 0.000 \\
\hline $40-49$ & $1208(35.8)$ & $576(47.7)$ & 18.56 & $8.11-42.45$ & 0.000 \\
\hline $50-59$ & $665(19.7)$ & $378(56.8)$ & 26.65 & $11.49-61.80$ & 0.000 \\
\hline $60 \&$ above & $560(16.6)$ & $280(50.0)$ & 37.74 & $15.97-89.16$ & 0.000 \\
\hline \multicolumn{6}{|l|}{ Religion } \\
\hline Christian & $218(6.5)$ & $66(30.3)$ & Ref. & & \\
\hline Hindu & $3021(89.6)$ & $1476(48.9)$ & 1.10 & $0.77-1.56$ & 0.595 \\
\hline Islam & $133(3.9)$ & $64(48.1)$ & 0.92 & $0.51-1.65$ & 0.787 \\
\hline \multicolumn{6}{|l|}{ Education status } \\
\hline None & 1396 (41.4) & $479(34.3)$ & Ref. & & \\
\hline 1-8 standard & $678(20.1)$ & $304(44.8)$ & 1.38 & $1.08-1.78$ & 0.010 \\
\hline 9-12 standard & $915(27.1)$ & $582(63.6)$ & 2.95 & $2.25-3.86$ & 0.000 \\
\hline College/university & $383(11.4)$ & $241(62.9)$ & 3.44 & $2.33-5.05$ & 0.000 \\
\hline \multicolumn{6}{|l|}{ Occupation } \\
\hline Skilled labour & $1152(34.2)$ & $388(33.7)$ & Ref. & & \\
\hline Farmer & $97(2.9)$ & $49(50.5)$ & 1.31 & $0.79-2.16$ & 0.283 \\
\hline House maker & $669(19.8)$ & $591(88.3)$ & 15.24 & $10.73-21.65$ & 0.000 \\
\hline Business & $187(5.5)$ & $133(71.1)$ & 1.83 & $1.20-2.81$ & 0.005 \\
\hline Govt. service & $249(7.4)$ & $176(70.7)$ & 2.38 & $1.54-3.66$ & 0.000 \\
\hline Self employed & $60(1.8)$ & $42(70.0)$ & 1.53 & $0.76-3.09$ & 0.231 \\
\hline Other & $958(28.4)$ & $227(23.7)$ & 0.38 & $0.29-0.50$ & 0.000 \\
\hline \multicolumn{6}{|l|}{ Car } \\
\hline No & $3152(93.5)$ & $1444(45.8)$ & Ref. & & \\
\hline Yes & $220(6.5)$ & $162(73.6)$ & 1.11 & $0.72-1.70$ & 0.631 \\
\hline \multicolumn{6}{|l|}{ Motorcycle } \\
\hline No & $2297(68.1)$ & $884(38.5)$ & Ref. & & \\
\hline Yes & $1075(31.9)$ & $722(67.2)$ & 1.24 & $0.97-1.59$ & 0.074 \\
\hline \multicolumn{6}{|l|}{ Television } \\
\hline No & $656(19.5)$ & $284(43.3)$ & Ref. & & \\
\hline Yes & $2716(80.5)$ & $1322(48.7)$ & 1.10 & $0.86-1.41$ & 0.418 \\
\hline \multicolumn{6}{|l|}{ Other land/property } \\
\hline No & $2847(84.4)$ & $1281(45.0)$ & Ref. & & \\
\hline Yes & 525 (15.6) & $325(61.9)$ & 0.94 & $0.72-1.23$ & 0.687 \\
\hline \multicolumn{6}{|l|}{ Computer } \\
\hline No & $3185(94.5)$ & $1445(45.4)$ & Ref. & & \\
\hline Yes & $187(5.5)$ & $161(86.1)$ & 3.69 & $2.10-6.47$ & 0.000 \\
\hline \multicolumn{6}{|l|}{ Hypertension (family history) } \\
\hline No & $2941(87.2)$ & $1362(46.3)$ & Ref. & & \\
\hline Yes & $431(12.8)$ & $244(56.6)$ & 1.20 & $0.89-1.60$ & 0.216 \\
\hline \multicolumn{6}{|l|}{ Diabetes (family history) } \\
\hline No & $2972(88.1)$ & $1301(43.8)$ & Ref. & & \\
\hline Yes & $400(11.9)$ & $305(76.2)$ & 3.18 & $2.23-4.54$ & 0.000 \\
\hline \multicolumn{6}{|l|}{ Tobacco user } \\
\hline Never used & $1251(37.1)$ & $730(58.4)$ & Ref. & & \\
\hline Formerly used & $216(6.4)$ & $102(47.2)$ & 0.85 & $0.57-1.27$ & 0.447 \\
\hline Currently user & $1905(56.5)$ & $774(40.6)$ & 1.04 & $0.82-1.33$ & 0.699 \\
\hline \multicolumn{6}{|l|}{ Consumed alcohol } \\
\hline No & $1871(55.5)$ & $1034(55.3)$ & Ref. & & \\
\hline Yes & $1501(44.5)$ & $572(38.1)$ & 0.93 & $0.74-1.15$ & 0.515 \\
\hline \multicolumn{6}{|l|}{ Financial stress } \\
\hline Moderate or severe & $1638(49.9)$ & $748(44.4)$ & Ref. & & \\
\hline Little or none & $1689(50.1)$ & $858(50.8)$ & 1.18 & $0.94-1.47$ & 0.145 \\
\hline Felt stress in last year & & & & & \\
\hline Never or some periods & $2560(75.9)$ & $1193(46.6)$ & Ref. & & \\
\hline Several periods or permanent stress & $812(24.1)$ & $413(50.9)$ & 1.85 & $1.45-2.35$ & 0.000 \\
\hline $\begin{array}{l}\text { Past } 12 \text { months, was ever you felt sad, } \\
\text { for } 2 \text { weeks or more in a row }\end{array}$ & & & & & \\
\hline No & $2858(84.8)$ & $1293(45.2)$ & Ref. & & \\
\hline Yes & $514(15.2)$ & $313(60.9)$ & 1.12 & $0.85-1.48$ & 0.403 \\
\hline Active at work & & & & & \\
\hline Heavy physical labour & $604(17.9)$ & $228(37.7)$ & Ref. & & \\
\hline Moderate work & $1251(37.1)$ & $599(47.9)$ & 1.45 & $1.11-1.90$ & 0.006 \\
\hline Subject does not work & $1517(45.0)$ & 779 (51.4) & 0.87 & $0.64-1.19$ & 0.416 \\
\hline
\end{tabular}




\begin{tabular}{|c|c|c|c|c|c|}
\hline Variable & $N(\%)$ & PR (\%) & AOR & $95 \% \mathrm{CI}$ & $p$-value \\
\hline \multicolumn{6}{|l|}{ Meat } \\
\hline$<3$ times/week & 3159 (93.7) & $1457(46.1)$ & Ref. & & \\
\hline$\geq 3$ times/week & $213(6.3)$ & 149 (70.0) & 1.50 & $0.98-2.29$ & 0.062 \\
\hline \multicolumn{6}{|l|}{ Fish } \\
\hline$<3$ times/week & $2305(68.4)$ & $1048(45.5)$ & Ref. & & \\
\hline$\geq 3$ times/week & 1067 (31.6) & $558(52.3)$ & 0.90 & $0.74-1.10$ & 0.322 \\
\hline \multicolumn{6}{|l|}{ Egg } \\
\hline$<3$ times/week & 3152 (93.5) & $1455(46.2)$ & Ref. & & \\
\hline$\geq 3$ times/week & $220(6.5)$ & $151(68.6)$ & 1.49 & $1.00-2.23$ & 0.047 \\
\hline \multicolumn{6}{|l|}{ Dairy products } \\
\hline$<3$ times/week & $3147(93.3)$ & $1432(45.5)$ & Ref. & & \\
\hline$\geq 3$ times/week & $225(6.7)$ & $174(77.3)$ & 2.57 & $1.68-3.94$ & 0.000 \\
\hline \multicolumn{6}{|l|}{ High energy food } \\
\hline$<3$ times/week & 3097 (91.8) & $1424(46.0)$ & Ref. & & \\
\hline$\geq 3$ times/week & $275(8.2)$ & $182(66.2)$ & 1.19 & $0.84-1.69$ & 0.307 \\
\hline \multicolumn{6}{|l|}{ Desserts/sweet } \\
\hline$<3$ times/week & $3029(89.8)$ & $1400(46.2)$ & Ref. & & \\
\hline$\geq 3$ times/week & $343(10.2)$ & $206(60.1)$ & 1.15 & $0.84-1.58$ & 0.363 \\
\hline \multicolumn{6}{|l|}{ Nuts/seeds } \\
\hline$\geq 3$ times/week & $934(27.7)$ & 366 (39.2) & Ref. & & \\
\hline$<3$ times/week & $2438(72.3)$ & $1240(50.9)$ & 2.37 & $1.90-2.96$ & 0.000 \\
\hline
\end{tabular}

doctor is not competent to tackle, $2.7 \%$ told that family members did not allow to have a health check-up.

Place for seeking treatment: Government healthcare was preferred by $61.6 \%$, whereas $32.7 \%$ prefer private clinic, $1.5 \%$ preferred private medical store/over the counter medicine and $4.2 \%$ preferred traditional system of medicine.
Reason for choosing particular place for treatment: Was proximity by $54.8 \%$, while $23.4 \%$ choose due to affordability and $21.8 \%$ choose as treatment was better.

Knowledge about previous diabetic status: Amongst diabetics $86.1 \%$ know about their status while $13.9 \%$ do not know about previous status.

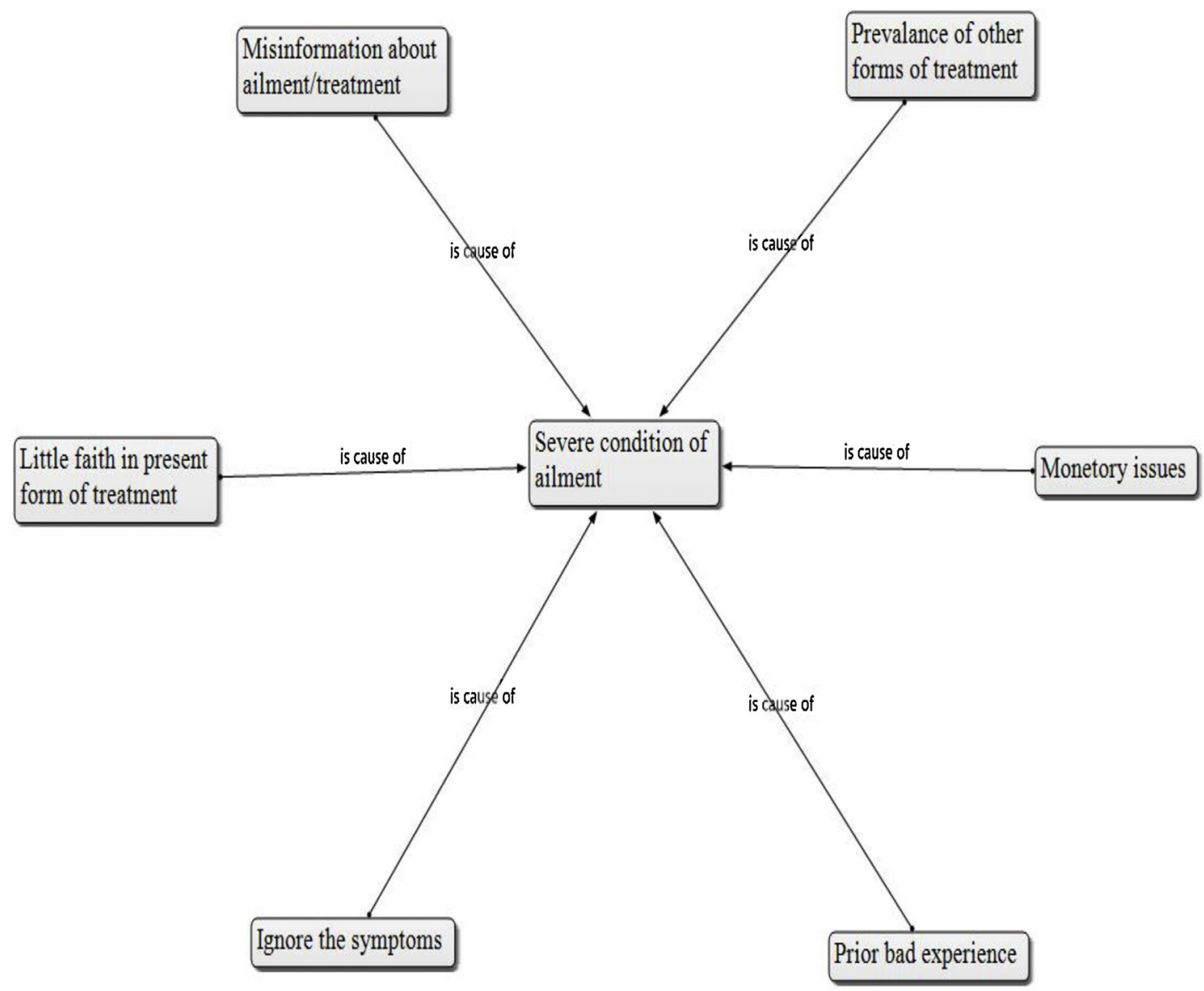

Fig. 1 - Schematic diagram showing the barrier affecting compliance of treatment. 
Time of detection of diabetes: Amongst known diabetics $51.6 \%$ detected as diabetic since 5 or more years while $32.3 \%$ detected within last 1-5 years and $16.1 \%$ detected as diabetic within last 1 year.

Treatment history: Among those having indication for use of statin was not utilized as treatment in $99.5 \%$, only $0.5 \%$ people use various statins as treatment. Among people who utilize statins, Atorvastatin (96.5\%) is mostly used followed by Rosuvastatin (3.5\%). Commonest dosage was $5 \mathrm{mg} /$ daily. Diuretic treatment was non-prevalent and so were other forms of treatment viz. ARB, ACE and antiplatelet. Insulin treatment was also non-prevalent. Beta-blocker treatment was complied by $6.2 \%$ and Ca channel blocker treatment in $9.2 \%$. Oral hypo-glycemics was utilized by $84.1 \%$ making it the most prevalent form of treatment. Amongst diabetics $36.1 \%$ were not taking any medicine. Blood testing was done by $69.4 \%$ to know their diabetic status while $30.3 \%$ do not do blood testing regularly. Blood test was done in a monthly interval by $28 \%$, quarterly interval in $12 \%$, half yearly in $8 \%$, annually in $8 \%$, more frequently in $16 \%$ and never in $28 \%$ amongst diabetics.

\section{Discussion}

MetS was common in $47.6 \%$, more common amongst men than women and was higher in well educated people and those hailing from higher economic status. Gender difference in access to medical care co-exist both in India and other developing countries. ${ }^{14}$ Hypertension was found in $62.2 \%$ cases of MetS whereas diabetes was present in $89.1 \%$ with MetS. Family history of hypertension and diabetes show significant association with MetS. Tobacco consumption, alcohol consumption, financial stress, depression and less PA were significantly associated with MetS. Three times or more consumption of meat, fish, egg, diary product, highenergy food, sweets/deserts and nuts and oilseed consumption were significantly associated with MetS.

Globally tobacco was the leading cause of morbidity and mortality, responsible for $18 \%$ deaths in high-income countries, $11 \%$ in middle-income countries, and $4 \%$ in lowincome countries. ${ }^{15}$ However, with smoking prevalence falling in high-income countries but still increasing in many middle and low income countries, the global burden from tobacco will shift increasingly from higher to lower income countries.

Multiple logistic regression analysis shows increasing age, education status, occupation, computer owner, persons with family history of diabetes, persons feeling stressed in last year, moderate PA, those consuming dairy product $\geq 3$ times/week and nuts and oilseed consumption of $<3$ times/week significantly associated with MetS. Several epidemiological studies demonstrated hypertension as independent risk factor for development of T2DM. Furthermore hypertension and diabetes often co-exists in same individual. ${ }^{16,17}$

The relationship between hypertension and T2DM is complex. Hypertension may exert impact upon the onset of T2DM through increasing vasoconstriction and vascular rarefaction, reducing skeletal muscle insulin sensitivity and detecting post receptor insulin signalling. ${ }^{18}$ A positive feedback link between hypertension and diabetes can facilitate onset of T2DM and worsen manifestation of each other. ${ }^{19}$ Hypertension and T2DM share many contributing behavioural and biological risk factors such as lack of PA, too much sedentary time and high dense energy food intake.

Qualitative study revealed most study participants (78.8\%) preferred allopathic medicine whereas Ayurvedic treatment was sought by $14.7 \%$ and $6.5 \%$ sought homoeopathic treatment. Distance to health care facility, expensive services, incompetent health care provider, lack of awareness, less priority given by family member were common reason for non-compliance, while government health care facility was the preferred place for seeking care amongst those compliant, although a sizable proportion who can afford prefers doing to private practitioner. ${ }^{20,21}$

Factor influencing place of treatment was distance or accessibility, affordability and quality of care. Regular blood testing was not done by $30.3 \%$. Oral hypo-glycemics is the most prevalent form of treatment amongst diabetic subjects with MetS, while use of statin is very poor. The risk of CVD is significantly higher in patients with T2DM (with or without hypertension) and hypertensive controls compared to healthy controls. ${ }^{22}$

Our study revealed physical inactivity as one risk factor of MetS. Recent reports have equated impact of PA to smoking with respect to worldwide burden of non-communicable diseases (NCDs). ${ }^{23}$ Physical activity is a challenging variable to measure due to inherent complexity and diversity of human behaviour. Clinical trials showing intervening with lifestyle modification or medications for individuals at high risk for hypertension may delay onset of hypertension. ${ }^{24}$

Indian Government launched National Program for Prevention \& Control of Cancer, Diabetes, Cardiovascular Diseases and Stroke (NPCDCS) to address high prevalence of NCDs. ${ }^{25}$ Risk factor control requires multidisciplinary approach, which includes approaching social determinants of health, health-care financing, improving medical education and health system strengthening. ${ }^{26}$

There is a need to do facility assessment to know the facility gap in provision for CVD care and to improve the quality of care and utilization of services. Provision of outreach services at regular interval can also be planned to address distance issue. Our assessment of depression and anxiety symptoms was based on self-report of symptoms using validated questionnaire and not on the Diagnosis and Statistical Manual (DSM) criteria based on clinical diagnosis interview. Second limitation may be recall bias expected in dietary assessment.

\section{Conclusion}

There is greater prevalence of MetS along with hypertension and diabetes. Behavioural risk factors such as tobacco use, low fruits and vegetables intake and high visible fat intake are higher. Right approach towards curbing the emergence of hypertension and diabetes amongst person with MetS may be to keep them under surveillance with application of all levels of prevention as and when needed. 


\section{Conflicts of interest}

The authors have none to declare.

\section{R E F E R E N C E S}

1. Reaven GM. Insulin resistance, the insulin resistance syndrome, and cardiovascular disease. Panminerva Med. 2005;47(4):201-210.

2. Bjorntorp P. Abdominal obesity and the metabolic syndrome. Ann Med. 1992;24(6):465-468.

3. National Institutes of Health. The Practical Guide to the Identification, Evaluation and Treatment of Overweight and Obesity in Adults. Bethesda: NIH; 2000.

4. Executive Summary of the Third Report of the National Cholesterol Education Program (NCEP): Expert Panel on Detection, Evaluation, and Treatment of High Blood Cholesterol in Adults (Adult Treatment Panel III). JAMA. 2001;285:2486-2497.

5. World Health Organization. International Association for the Study of Obesity and International Obesity Task Force: The Asia Pacific Perspective: Redefining Obesity and its Treatment. Sydney: Health Communications Australia Pty Limited; 2000:17-21.

6. Mahanta TG, Joshi R, Mahanta BN, Xavier D. Prevalence of modifiable cardiovascular risk factors among tea garden and general population in Dibrugarh, Assam, India. J Epidemiol Global Health. 2013;3:147-156.

7. Grundy SM, Cleeman JI, Daniels SR, et al. Diagnosis and management of the metabolic syndrome: an American Heart Association/National Heart, Lung, and Blood Institute Scientific Statement. Circulation. 2005;112:2735-2752. http:// dx.doi.org/10.1161/CIRCULATIONAHA.105.169404.

8. Hui WS, Liu Z, Ho SC. Metabolic syndrome and all-cause mortality: a meta-analysis of prospective cohort studies. Eur J Epidemiol. 2010;25(6):375-384. http://dx.doi.org/10.1007/ s10654-010-9459-z.

9. Sarrafzadegan N, Kelishadi R, Baghaei A, et al. Metabolic syndrome: an emerging public health problem in Iranian Women: Isfahan Healthy Heart Program. Int J Cardiol. 2008;131:90-96. http://dx.doi.org/10.1016/j.ijcard.2007.10.049.

10. Misra A, Khurana L. Obesity and the metabolic syndrome in developing countries. J Clin Endocrinol Metab. 2008;93:S9-S30. http://dx.doi.org/10.1210/jc.2008-1595.

11. Galassi A, Reynolds K, He J. Metabolic syndrome and risk of cardiovascular disease: a meta-analysis. Am J Med. 2006;119:812-819. http://dx.doi.org/10.1016/j. amjmed.2006.02.031.

12. Gupta R, Deedwania PC, Sharma K, et al. Association of educational, occupation and socioeconomic status with cardiovascular risk factors in Asian Indians: a crosssectional study. PLoS One. 2012;7(August (8)):e44098.
13. District Health Action Plan 2014. Dibrugarh district of Assam, 11-12.

14. Pierce JP, Choi WS, Gilpin EA, Farkas AJ, Berry CC. Tobacco industry promotion of cigarettes and adolescent smoking. JAMA. 1998;279(7):511-515.

15. Bajra SJ, Sehgal V, Kalra S, Baruah MP. Management of diabetes mellitus type 2 in geriatric population: current perspectives. J Pharm Bioallied Sci. 2014;6:151-157.

16. Przewozny T, Gojska-Grymajto A, Kwarciany M, Gasecki D, Narkiewicz K. Hypertension and cochlear hearing loss. Blood Press. 2015;24:199-205.

17. Weycker D, Nichols GA, O'Keeffe-Rosetti M, et al. Excess risk of diabetes in persons with hypertension. J Diabetes Complications. 2009;23:330-336.

18. Chobanian AV, Bakris GL, Black HR, et al. Seventh report of joint national committee on prevention, detection, evaluation and treatment of high blood pressure. Hypertension. 2003;42:1206-1252.

19. Cheung BMY. The hypertension-diabetes continuum. J Cardiovasc Pharmacol. 2010;55:333-339.

20. Lonati C, Morganti A, Comarella L, Mancia G, Zanchetti A. Prevalence of type 2 diabetes amongst patients with hypertension under the care of 30 Italian clinics of hypertension: results of the (Iper)tension and (dia)bete study. J Hypertension. 2008;26:1801-1808.

21. The Diabetes Control and Complication Trial Research Group. The effect of intensive treatment of diabetes on the development and progression of long term complications in insulin dependent diabetes mellitus. $N$ Engl J Med. 1993;329:977-986.

22. Khunti K, Gray IJ, Skinner T, et al. Effectiveness of the diabetes education and self-management for ongoing and newly diagnosed (DESMOND) for people with newly diagnosed type 2 diabetes: three year follow up of a cluster randomized clinical trial in primary care. BMJ. 2012;344: e2333.

23. Kulkarni NB, Vedak TK, Ganu MU, Godbole SG, Deo SS. Assessment of cardiometabolic risk factors and its association with Framingham risk score in Indian patients with type 2 diabetes mellitus. Int J Diabetes Dev Ctries. 2015;35 (5):340-345.

24. Ministry of Health and Family Welfare, Government of India. Annual Report to the People on Health 2014-15. http:// www.mohfw.nic.in/WriteReadData/1892s/ 7896321456325698.pdf. Accessed 19 December 2015.

25. Gupta R, Guptha S, Joshi R, Xavier D. Translating evidence into policy for cardiovascular disease control in India. Health Res Policy Syst. 2011;9:8. http://dx.doi.org/10.1186/1478-45059-8.

26. Subramanian SV, Corsi DJ, Subramanyam MA, Smith GD. Jumping the gun: the problematic discourse on socioeconomic status and cardiovascular health in India. Int J Epidemiol. 2013;42(5):1410-1426. 